San Jose State University

SJSU ScholarWorks

Master's Theses

Master's Theses and Graduate Research

1993

\title{
The Effects of applied periodic sound on the circadian timing system of the Sprague-Dawley rat
}

Karen Audrey Moeller

San Jose State University

Follow this and additional works at: https://scholarworks.sjsu.edu/etd_theses

\section{Recommended Citation}

Moeller, Karen Audrey, "The Effects of applied periodic sound on the circadian timing system of the Sprague-Dawley rat" (1993). Master's Theses. 562.

DOI: https://doi.org/10.31979/etd.vkn7-8h2f

https://scholarworks.sjsu.edu/etd_theses/562

This Thesis is brought to you for free and open access by the Master's Theses and Graduate Research at SJSU ScholarWorks. It has been accepted for inclusion in Master's Theses by an authorized administrator of SJSU ScholarWorks. For more information, please contact scholarworks@sjsu.edu. 


\section{INFORMATION TO USERS}

This manuscript has been reproduced from the microfilm master. UMI films the text directly from the original or copy submitted. Thus, some thesis and dissertation copies are in typewriter face, while others may be from any type of computer printer.

The quality of this reproduction is dependent upon the quality of the copy submitted. Broken or indistinct print, colored or poor quality illustrations and photographs, print bleedthrough, substandard margins, and improper alignment can adversely affect reproduction.

In the unlikely event that the author did not send UMI a complete manuscript and there are missing pages, these will be noted. Also, if unauthorized copyright material had to be removed, a note will indicate the deletion.

Oversize materials (e.g., maps, drawings, charts) are reproduced by sectioning the original, beginning at the upper left-hand corner and continuing from left to right in equal sections with small overlaps. Each original is also photographed in one exposure and is included in reduced form at the back of the book.

Photographs included in the original manuscript have been reproduced xerographically in this copy. Higher quality $6 "$ " 9 9" black and white photographic prints are available for any photographs or illustrations appearing in this copy for an additional charge. Contact UMI directly to order.

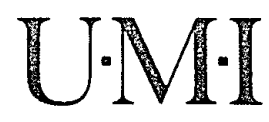



Order Number 1853039

The effects of applied periodic sound on the circadian timing system of the Sprague-Dawley rat

Moeller, Karen Audrey, M.A.

San Jose State University, 1993

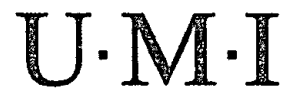

300 N. Zeeb Rd.

Ann Arbor, MI 48106 

THE EFFECTS OF APPLIED PERIODIC SOUND ON THE CIRCADIAN TIMING SYSTEM OF THE SPRAGUE-DAWLEY RAT

\author{
A Thesis \\ Presented to \\ The Faculty of the Department of Biological Sciences \\ San Jose State University
}

In partial fulfillment

of the Requirements for the Degree

Master of Arts

By

Karen Audrey Moeller

May, 1993 
APPROVED FOR THE DEPARTMENT OF BIOLOGICAL SCIENCES $\frac{\text { Lan follil }}{\text { Daniel C. Hojley, Rh.D. }}$ Professor, San Jose State University

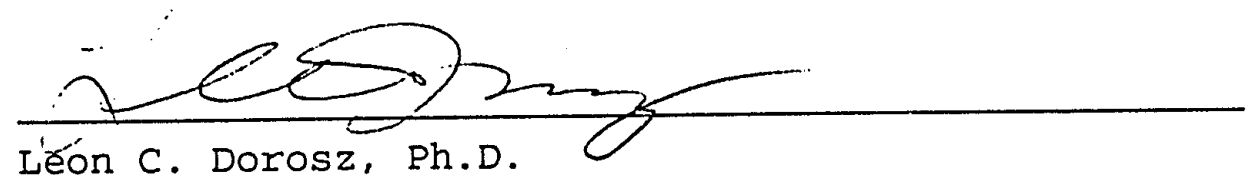
Professor, San Jose State University

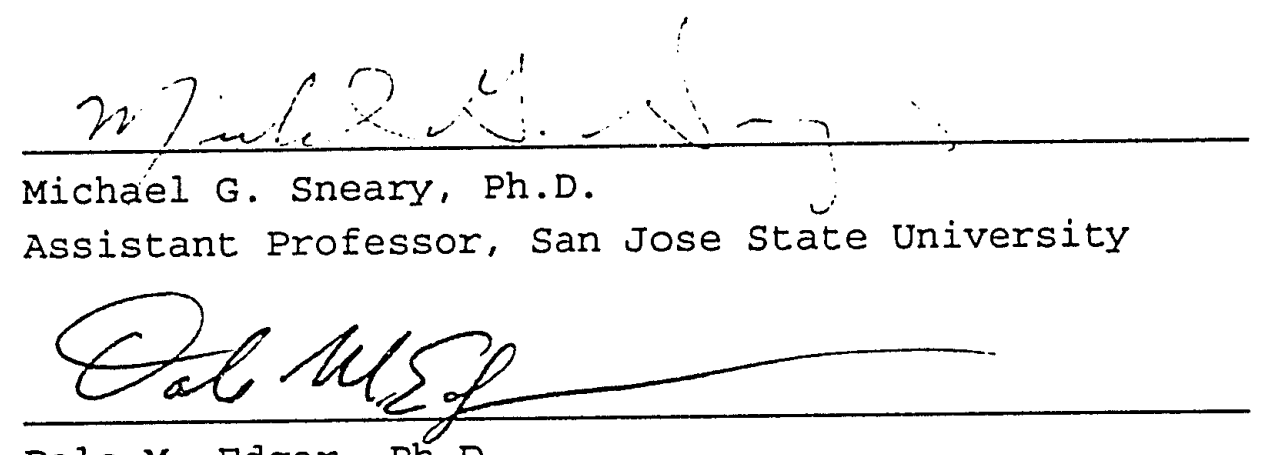

Dale M. Edgar, Ph.D.

Sr. Research Scientist, Dept.of Psychiatry \& Behavioral Sciences, Stanford University School of Medicine

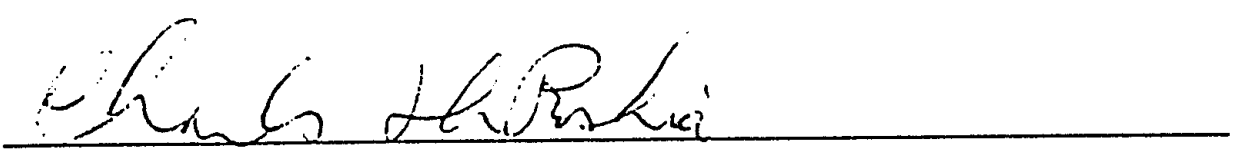

Charles W. DeRoshia, M.A. Research Scientist, NASA-Ames Research Ctr.

APPROVED FOR THE UNIVERSITY Herexe Or. Stanfore 


\section{ABSTRACT}

We examined whether regularly scheduled white noise (N) (80$90 \mathrm{~dB})$ could synchronize or influence the free-running drinking, feeding, and locomotor circadian rhythms of male sprague-Dawley rats. Individually housed animals were entrained to a light-dark cycle (12L:12D), with no sound (Q), followed by constant darkness (DD1). Subsequently, a noise regimen (12N:12Q) was administered for 60 days (minimum). A second population of rats was similarly treated, but exposed to a $4 \mathrm{~N}: 20 \mathrm{Q}$ cycle. Both populations were finally exposed to a DD2 epoch. All rats from the first population and $1 / 3$ of rats from the second population exhibited significantly shortened periods during DD2 relative to periods during DD1. The noise influence on rhythm period was not attributable to "masking effects," as verified in a third study. Our findings indicate that periodic noise influences, but does not entrain, the circadian rhythm of rats. 


\section{ACKNOWLEDGEMENTS}

This research project resulted from the combined efforts by many individuals. First, I would like to express my sincere thanks and gratitude to Dr. Daniel C. Holley for not only serving as my graduate advisor, but for his support, encouragement, and confidence in my abilities throughout this endeavor. Special thanks to Charles W. DeRoshia, NASA-Ames Research Center, for the generous use of his computer facilities, for his constructive criticism and for sharing his vast knowledge of data analysis with me. Thanks to Dr. Dale M. Edgar, Stanford University, whose critical discussions of experiments and procedures were invaluable. I thank Dr.'s Leon C. Dorosz and Micheal G. Sneary, San Jose State University, for their encouragement and for their time reading and critiquing this thesis. Thanks to Dr. Tim Rohm for generously providing the sound measuring equipment. Special thanks and gratitude to Gary D. Mele for his endless support in every aspect of this project. I would also like to acknowledge the following susu students for their excellent technical assistance: Jill A. Erickson, Mark P. Robbins, Mono Nong, and Heather Hinchcliffe. Special thanks to Nathan Norris for his support, understanding, and friendship without which this project would have been far more difficult. Thanks to Mom, Dad, \& Eric for their constant encouragement and support. Last, but not least, I would like to thank Dr. H. W. Colvin, Jr. for introducing 
me to Dr. Holley, for encouraging me to pursue a graduate degree, and for always sharing his wisdom and encouragement.

This study was funded by NASA Cooperative Agreement NCC2-593, between D.C. Holley, San Jose State University and NASA-Ames Research Center. 


\section{INTRODUCTION}

Most physiological and behavioral parameters in animals exhibit rhythms with a period of about 24 hours. These circadian rhythms normally synchronize to various periodic environmental stimuli. However, when an animal is subjected to controlled conditions where environmental variables are held constant le.g. light, food availability, temperature, and sound the animal will generate cellular and overt rhythms with periods that differ slightly from 24 hours. These rhythms are referred to as freerunning and display non-stationary phase angles with regard to sidereal time (Aschoff, 1960).

Photic and non-photic environmental variables have been categorized according to their ability to synchronize physiological and behavioral rhythms; however, light/dark (LD) cycles, are thought to be the dominant environmental zeitgeber entraining biological rhythms in most species, including man (Aschoff et al., 1982; Bruce, 1960; Czeisler et al., 1981). Nonphotic zeitgebers including food availability cycles (Aschoff et al., 1983; Edmonds \& Adler, 1977; Mistlburger et al., 1990; Sulzman et at., 1977; Rusak, 1989), environmental temperature fluctuations (Sweeney, 1960; Lindberg \& Hayden, 1974), barometric pressure changes (Hayden, 1969), electrostatic field cycles (Dowse, 1969) and scheduled volitional exercise (Edgar \& Dement, 1991) are reported to synchronize circadian rhythms in various 
species.

In addition to the non-photic zeitgebers mentioned above, social interactions are thought to systematically influence the circadian system. Social time cues (visual, acoustical, pheromonal, etc.) have been shown to function as zeitgebers in bats (Marimuthu \& Chandreshakaran, 1978, 1979, 1981), mice (Mus musculus), antelope, wolf-coyote hybrid, beaver (Castor canedensis) colonies, macaque monkeys (Chandreshakaran, 1982), golden hamsters (Mesocricetus auratus) (Mrosovsky, 1988), and deer mice (Peromyscus maniculatus) (Crowley et al., 1980). However, similar studies performed on the common marmoset (Callithrix jacchus) (Erkert et al., 1986), Australian sugar glider (Petaurus breviceps) (Kleinknecht, 1985), and the laboratory rat (Borbély, 1982 all failed to show social entrainment. Research using humans has indicated that social factors may act as circadian rhythm zeitgebers in man (wever, 1979; winget et al., 1989; Aschoff, 1971).

Studies that have investigated sound as a zeitgeber suggest that acoustical disturbances can entrain birds (Gwinner, 1966; Menaker \& Eskin, 1966; Lohman \& Enright, 1967; Reebs, 1989), golden hamsters (Mesocricetus auratus) (Meyer, 1968), and cats (Felis catus L.) (Randall, 1990) but fail to entrain the squirrel monkey (Saimiri sciureus) (Sulzman et al., 1977).

In the controlled environment of the proposed U.S. Space station Freedom, animals will be maintained and studied for extended periods. Simultaneously, crews may have various work- 
rest schedules which produce periodic noises. The present study was undertaken to established whether sound or social noises influence the circadian system of the Sprague-Dawley rat. Our findings suggest that periodic noise does not entrain drinking, feeding, or locomotor activity (LMA) rhythms, but may influence the stability of the free-running circadian period. 
MATERIALS \& METHODS

Animals and apparatus

Male Sprague-Dawley rats, purchased from Simonsen Laboratories (Gilroy, CA) weighing approximately $150 \mathrm{~g}$ at the start of the experiment, were housed individually in Nalgene metabolic cages (Nalge Co., Rochester, NY). The cages were placed inside separate ventilated, radio frequency shielded, wooden hives (I.D. $66 \mathrm{~cm} \times 66 \mathrm{~cm} \times 76 \mathrm{~cm}$ ). These hives were contained in a sound attenuated, environmental chamber at constant temperature (20-22 $\left.C^{\cdot}\right)$. Light was provided to each hive via broad spectrum fluorescent lights (Vita-Lites, Duratest Corp., Fairfield, NJ ) with an intensity of $5.0 \pm 1.0$ (SD) Iux measured at the height of the animal's head when standing. Light measurements were taken with a calibrated IL-1700 Research Radiometer (International Lighting Co., Newburyport, MA).

The amplified sound used during this study was produced by a Synthi-AKS (EMS Ltd., London, UK) sound generator with a maximum frequency of $10 \mathrm{kHz}$ and was distributed to each hive through individual speakers (Radio Shack \#40-1248B, Tandy Corp., TX). Though the white noise function was used, the measured decibel intensity at each octave band frequency $(31.5 \mathrm{~Hz}-8000 \mathrm{~Hz})$ ranged from $70 \mathrm{~dB}$ to $92 \mathrm{~dB}$. Therefore, the projected noise should be considered indiscriminate and not pure "white" noise. The sound 
intensities were adjusted to 80-90 dB (See Protocols) with a Quest \#215 sound level meter and octave band intensities were measured with a Quest octave band analyzer (Quest Electronics, Oconomowoc, WI).

The behavioral rhythms monitored were locomotor activity (LMA), drinking activity, and feeding activity. LMA was recorded using a three-axis accelerometer (straindyne Eng. Co., Los Altos, CA) which was attached to the cage stand. The stand was positioned atop a plexiglas platform supported by semi-rigid springs which allowed omnidirectional cage movement in response to animal activity. The accelerometer transduces the physical motion of the cage to a voltage that is proportional to the cage displacement (displacement units). This voltage was then digitized and recorded. Drinking activity was recorded by the completion of an electrical circuit that ran from the metal grid cage bottom to the water lick. When the rat drank, the circuit was completed and duration of drinking activity was recorded. water was available ad libitum. Feeding activity was recorded via an infrared light beam located above the food tray (Model \#FM8, Omnitech Electronics, Inc., Columbus, $O H)$. When the rat interrupted the beam, the monitoring system recorded duration of feeding activity. Throughout all phases of the investigation food (Wayne Rodent BLOX 8604) was available ad libitum.

All monitored activities (locomotor activity, feeding, and drinking) were recorded using a Keithley Data Acquisition System (DAS) located in a room separate from the experimental chamber. 
Behavioral events were summed and recorded every ten minutes. Animal maintenance was performed on alternate days throughout the experiment to minimize disturbances of the animal's circadian system. During the constant dark (DD) epochs of the study animal maintenance was performed under a red light filter (Roscolux Roscoesun \#27 Medium Red, Musson Theatrical, Santa Clara, CA) with $50 \%$ cutoff at 660 nanometers; $4 \%$ transmission.

Data Analysis

Data collected from all experiments were transferred from the Keithley (MS-DOS based) data acquisition system to a Hewlett Packard 9836 computer for editing, filtering, and time series analysis. Raw data for all parameters was initially processed using a box-and-whisker (Tukey, 1977) editing program which objectively removed outliers, editing an average of $1.04 \pm$ 1.26(SD)\% of the data. The feeding and drinking values were then converted from 10 minute sample point values to numbers of nonzero 10 minute sample point values per each one hour bin. The locomotor activity (LMA) data were reduced through decimation. This procedure converts six 10 minute recordings into hourly means.

The data then underwent robust locally weighted regression (RLWR), a non-parametric detrending process (Cleveland, 1979). This filter ( 72 terms, filters data $>3$ cycles) removes low frequency rhythms or trends which may obscure periodicities of 
interest. After editing and filtering, the data were subjected to periodicity analysis.

To obtain an accurate estimation of phase and amplitude of the circadian rhythm during the various environmental conditions the data were folded out $20 \%$ on each end and then underwent complex demodulation (Sing, 1980). The period of the rhythm (Tau) for each experimental condition was calculated by performing a linear regression on the first determined acrophase (peak time of best fit sine function) of each cycle. The estimated period of the rhythm during each experimental epoch was confirmed by processing the detrended data through FFT spectral analysis (Bloomfield, 1976) with 5 day windows and 2 day moving increments. Experiment 3 data initially underwent $z$-score normalization (Zar, 1984) followed by a mean educed cycle procedure. The mean educed cycle averages the data for each rat across each day at each of the 144 ten minute sample points. This smooths the circadian rhythm and allows for visualization of phase-dependent masking effects which would produce peaks or depressions in response to the applied noise/quiet cycle.

Statistical significance of period, phase, and amplitude changes for the entrainment studies were determined through a repeated measures two-way analysis of variance (ANOVA). Significance of changes in activity levels during various conditions in experiment 3 were determined by a four-way repeated measures ANOVA. Null hypothesis was rejected for $p<.025$. Data are presented using mean $\pm S D$ unless otherwise indicated. 
Entrainment Experiments

Experiment 1:

Six rats were acclimated for 14 days to a 12L:12D photo period.

The average light intensity was $5.08 \pm 0.42$ lux. Following acclimation, the experiment began with a 6 day control period having 12L:12D lighting and no applied sound. Baseline ambient noise within the cages was $64.6 \pm 3.3 \mathrm{~dB}$. The baseline period was followed by an 8 day period of constant dark (DD-1; <0.01 Lux). After the DD-1 eight day period, the animals were subjected to a $12 \mathrm{~N}: 12 \mathrm{Q}$ cycle (noise/quiet) while they remained in DD conditions. The sound "on" period began at 19:00 and ended at 07:00. In order to avoid habituation to monotonous sound stimulation intermittent noise was chosen. The twelve hour sound "on" cycle therefore consisted of 2 minutes oN $(83.4 \pm 1.34 \mathrm{~dB})$ followed by 13 minutes OFF $(64.6 \pm 3.3 \mathrm{~dB})$. This schedule lasted for 60 days. Following this regimen, a 32 day DD freerun period (DD-2) was reestablished.

Experiment 1 Timeline:

Acclimation

Control (12L:12D)

DD-1 (freerun)

$\mathrm{DD}-\mathrm{N}: \mathrm{Q}$

$D D-2$ (freerun)
14 days

06 days

08 days

60 days

32 days 
Experiment 2:

The protocol for this experiment differs from the first in that the $2 \mathrm{~min}$. ON:13 min. OFF sound $(88 \pm 0.82 \mathrm{~dB})$ was applied in concentrated 4 hour periods ( $4 \mathrm{ON}$ : $20 \mathrm{OFF}$ ) starting at circadian time (CT) 10. This schedule was chosen based on Mrosovsky's (1988) non-photic phase response curve (PRC) (See Discussion). Circadian time (CT) is determined by taking the animals circadian cycle and dividing it into 24 equal parts. Each part represents an "hour." BY convention, CT 0 is defined as the onset of activity in diurnal animals and CT 12 is defined as the onset of activity in nocturnal animals. In order to expose the rats to sound during their CT 10 - CT 14 window, the rats were monitored during baseline in $\mathrm{DD}$ and their mean activity onset (CT 12) was determined. BY convention, CT 10 was defined as 2 hours prior to activity onset.

Light intensity was measured in the same manner as in all other experiments with the average intensity measuring $4.95 \pm .05$ lux. Light intensity was $<0.01$ lux in DD conditions. 
Experiment 2 Timeline:

$\begin{array}{lr}\text { Acclimation } & 10 \text { days } \\ \text { Control (12L:12D) } & 14 \text { days } \\ \text { DD-1 (freerun) } & 17 \text { days } \\ \text { DD-NQ* } & 69 \text { days } \\ \text { DD-2 (freerun) } & 11 \text { days }\end{array}$

Masking Experiment

Experiment 3 determined whether sound elicited masking effects in the monitored behavioral parameters. Rats were initially exposed to a 10 day acclimation period in LD. Following acclimation, the rats were monitored for 5 days in 12L:12D with no applied sound. This baseline period was followed by a 6 day 12L:12D light schedule accompanied with a 2 hour ON: 2 hour OFF (ambient) sound cycle. The applied sound cycle (N) was comprised of intermittent "white" noise with a 2 minute $\mathrm{ON}(89 \pm 0.58 \mathrm{~dB})$ : 13 minute OFF $(65.8 \pm 2.7 \mathrm{~dB})$ repeating schedule. By using a periodic sound regimen, any masking effects produced should appear clearly in the educed cycle.

Following the $12 \mathrm{~L}: 12 \mathrm{D}-\mathrm{N} / \mathrm{Q}$ epoch the rats were monitored for 7 days in 12L:12D schedule with no applied sound. Subsequently,

* The sound epoch incorporated the average CT 12 in the middle of the 4 hour administration. Specifically, activity onset was extrapolated to be approximately 21:15 at the end of the first DD epoch. In accordance with this estimation, the sound timer was set to turn on at $19: 15$ and turn off at $23: 15$. 
the rats were subjected to a $\mathrm{DD}-\mathrm{N} / \mathrm{Q}$ cycle. This routine was similar to the above mentioned noise:quiet cycling schedule and ran for 4 days. Transition of lights, ON/OFF, took place at 07:00 and 19:00, respectively. The sound cycling routine was turned oN at 19:00 on day 1 , and turned OFF at 19:00 four days later.

Experiment 3 Timeline:

Acclimation

Control (12L:12D)

N/Q - 12L:12D

Control (12L:12D)

$N / Q-D D$
10 days

05 days

06 days

07 days

04 days 


\section{RESULTS}

Entrainment studies

Figure 1 shows double raster plots of the three monitored parameters from a representative animal (animal 2, experiment 1). Entrainment of the rhythms is demonstrated during the LD cycle in all parameters. Upon release to constant conditions (DD1) the average free-running period significantly increased $(24.39 \pm 0.09$ $h$, ANOVA, $p<.025)$. In calculating the mean Tau, the period values estimated for rat \#4 (LMA) during the LD and DD1 epochs were not used because they were outliers, deviating from the mean by more than 3 standard deviations. All other data were used. The period from the 60 day sound window is represented in 20 day increments (DDNQ-1 = days $1-20$, etc.) (Tables $1 \& 2$ and Figures 3 \& 4) for a more accurate description of the period dynamics during that epoch. Within the first twenty days of the applied sound condition (DD-NQ1) the rhythm shows an initial lengthening followed by a shortening of the period which resulted in a "scalloping" appearance of the rhythm (See Fig. 1 a,b,c). Following these transients, Tau gradually decreased $0.38 \mathrm{~h}$ to an average period of $23.93 \pm .13 \mathrm{~h}$ in the last 20 days (DD-NQ3) of the sound epoch. The second freerun condition following the sound application continued to show a significant decrease in Tau (ANOVA, $\mathrm{p}<.025$ ) in all three parameters relative to the initial 
free-running period. The final freerun conditions resulted in an average Tau of $23.86+0.08 \mathrm{~h}$ for all rats, all parameters, which represents a $0.45 \mathrm{~h}$ decrease from the original freerun period (DD1) (Figure 2a,b). Table 1 shows the corresponding period lengths of each rat during all experimental conditions. The 60 day sound window was separated into 20 day increments (DDNQ-1 = days 1-20, etc.) for a higher resolution of the period changes during that epoch.

Figure 3 shows double raster plots from the drinking activity obtained from each animal in experiment 2 . Figure $3 b, c, d, \& f$ shows non-stationary circadian rhythms (with respect to phase and period) with similar patterns while Fig.3 a \& e show smaller changes in Tau over time. Table 2 shows the periods of the drinking activity corresponding with the raster plots displayed in Fig. 3. Estimated Tau from the feeding activity rhythm corresponded with the estimated Tau of the drinking activity rhythm but is not shown. Due to a technical malfunction, the LMA data for all rats was not available for this portion of the study. - All six rats in experiment 2 exhibited entrainment to the LD cycle. In freerun conditions (DD1), four of the six rats $(\# 2, \# 3, \# 4, \# 6)$ displayed an average Tau of $24.19 \pm 0.08 \mathrm{~h}$ for the drinking activity rhythm while the other two rats $(\# 1, \# 5)$ maintained period lengths of $24.04 \mathrm{~h}$ and $24.00 \mathrm{~h}$ for drinking, respectively. During the final freerun condition (DD2), drinking and feeding periods ranged from $23.66 \mathrm{~h}$ to $24.38 \mathrm{~h}$ and from $23.85 \mathrm{~h}$ to 24.48 , respectively. 
Figure $4(a, b, c)$ shows the mean period length, acrophase, and rhythm amplitude for the drinking parameter measured in experiments 1 and 2 . Though not presented, feeding activity and LMA showed similar results.

Figure $4 a$ offers a comparison of the average changes observed in Tau over the length of the two experiments. The graph indicates that experiment 1 rats demonstrated a free-running period that differed more from 24 hours than rats in experiment 2 . Experiment 2 results also differed from experiment 1 in that upon presentation of the sound, the rhythm period length continued to increase through the first twenty days of the sound schedule, while experiment 1 shows that the period had already begun to decrease during that time. From the first 20 days of the sound epoch up through the end of the sound epoch, the period from experiment 2 displayed a linear decrease while experiment 1 showed the primary decrease in period occurred between days 21-40 of the sound epoch and then decreased slowly in the final 29 days. The period lengths during the final freerun of the two protocols diverge. Experiment 1 shows that Tau continues to decrease while the mean period in experiment 2 increases back toward the initial freerun period.

Figure $4 \mathrm{~b}$ compares the mean acrophases of the drinking rhythms between the two experiments during all experimental conditions. A mean drinking acrophase of $264.6 \pm 3.3$ degrees was obtained during the LD entrainment period (Expt. 1). Corresponding to the increasing Tau during the DD1 free-running condition, the 
acrophase delayed to $281.8 \pm 5.9$ degrees. During the first 20 days of the sound epoch (N/Q 1), the acrophase advanced significantly (ANOVA, $p<.025$ ). This trend continued, for the next 20 days until the mean peak acrophase was $342.7 \pm 8.4$ degrees. The acrophase then displayed a marked delay during the final 20 days of the sound regimen to a mean of $326.8 \pm 22.4$ degrees. This trend continued through the 30 day freerun condition to a final mean acrophase of $301.3 \pm 24.2$ degrees. Experiment 2 displays trends similar to those represented by the first experiment except in the final phase during the last freerun condition (DD2). The mean acrophase for the LD entrainment condition was $255.9 \pm 8.5$ degrees. The initial free-running acrophases in this experiment ranged from 255.1 to 287.8 degrees. This range illustrates the variety of acrophases found in the individual rats (See Fig. 3). The mean acrophase showed a maximum delay between days 21-40 of the sound epoch. The delaying acrophase from DD1 to N/Q 2 displayed a slope that was greater than that seen during the free-running condition. The mean acrophase advanced during the last N/Q 3 window. Standard error for the mean during this time was large due to the fact that the rhythms of the individual rats varied markedly (Fig. 3). From the graph it is clear that phase control was not demonstrated by the zeitgeber in either protocol.

Figure $4 \mathrm{c}$ illustrates the trend of the rhythm amplitude over the course of the two experiments. The mean amplitudes from both experiments decrease at nearly the same rate from LD to DD1 
condition. Experiment 1 showed a linear decrease in the amplitude throughout the sound epoch, followed by a slight increase in amplitude during the final DD2 condition. Experiment 2 displayed a decrease in the drinking rhythm amplitude up through the first 40 days of the sound window (Fig 4C). The decrease, however, was not linear; amplitude dropped 0.46 units (number of events/hour) from DD1 to N/Q 1 , but only decreased by 0.09 units from $N / Q 1$ to N/Q 2. The last 29 days of the sound window (N/Q 3 ) showed a significant increase in the rhythm amplitude (ANOVA, $p<.025$ ) relative to the previous 20 days (N/Q 2). The total decrease of the mean rhythm amplitudes from the start to the end of both experiments are not statistically significant.

Masking Experiment

Figure 5 compares the mean LMA levels of the 12L:12D epoch with the $12 \mathrm{~L}: 12 \mathrm{D} ; 24 \mathrm{~N} / \mathrm{Q}$ regimen (See Methods). The mean amplitude for the group $(n=6)$ was determined by $z$-score normalization of the values.

The masking effects produced by the light cycle combined with the underlying circadian rhythm of the LMA are evident by the marked changes in the activity levels. In the dark, without the sound application, the mean activity level was $3223.83 \pm 1.51$ (S.E.M.) displacement units (d.u.) (See Methods). This value is significantly higher than the mean activity value, $3178.49 \pm 1.60$ (S.E.M.) d.u., found when the rats were exposed to light (ANOVA, 
$\mathrm{p}<.025)$. The mean activity level of the animals during 12L:12D, 2 hour "on" sound schedule, was $3190.89 \pm 1.64$ (S.E.M.) d.u.. The mean activity value for the 12L:12D no applied sound was $3194.08 \pm$ 1.65 (S.E.M.) d.u.; these values do not differ significantly. Had sound produced a masking effect, two hour peaks or depressions in the mean activity that correspond with the administered sound cycle would be evident in the educed cycle. 
Table 1. Experiment 1. Periods (hours) for each rat, during each experimental condition.

$\begin{array}{lccccccc}\text { Rat \# } & 1 & 2 & 3 & 4 & 5 & 6 & \text { mean } \pm \text { S.D } \\ \text { LD } & 23.87 & 24.00 & 23.83 & 24.07 & 23.65 & 23.83 & 23.88 \pm 0.14 \\ \text { DD1 } & 24.43 & 24.39 & 24.32 & 24.25 & 24.39 & 24.40 & 24.36 \pm 0.07 \\ \text { DD/NQ-1 } & 24.06 & 24.19 & 24.20 & 24.20 & 24.11 & 24.14 & 24.15 \pm 0.06 \\ \text { DD/NQ-2 } & 23.96 & 24.01 & 24.00 & 23.94 & 24.00 & 23.93 & 23.97 \pm 0.03 \\ \text { DD/NQ-3 } & 24.00 & 23.87 & 23.97 & 23.97 & 24.00 & 23.94 & 23.96 \pm 0.05 \\ \text { DD2 } & 23.96 & 23.84 & 23.92 & 23.77 & 23.82 & 23.87 & 23.86 \pm 0.07\end{array}$

Table 2. Experiment 2. Periods (hours) for each rat, during each experimental condition.

$\begin{array}{lccccccc}\text { Rat \# } & 1 & 2 & 3 & 4 & 5 & 6 & \text { mean } \pm \text { S.D } \\ \text { LD } & 24.00 & 23.93 & 23.93 & 23.88 & 23.97 & 24.00 & 23.95 \pm 0.05 \\ \text { DD1 } & 24.04 & 24.16 & 24.28 & 24.20 & 24.00 & 24.10 & 24.13 \pm 0.10 \\ \text { DD/NQ-1 } & 24.07 & 24.24 & 24.31 & 24.20 & 24.09 & 24.22 & 24.19 \pm 0.09 \\ \text { DD/NQ-2 } & 23.97 & 24.13 & 24.15 & 24.15 & 24.08 & 23.97 & 24.08 \pm 0.08 \\ \text { DD/NQ-3 } & 23.81 & 24.06 & 24.08 & 24.00 & 23.83 & 23.90 & 23.95 \pm 0.11 \\ \text { DD2 } & 24.38 & 23.72 & 24.15 & 23.66 & 24.38 & 24.24 & 24.09 \pm 0.32\end{array}$

Table $1 \& 2$. Tau ( $h$ ) for drinking activity rhythm across all experimental conditions. LD-12L:12D; DD1- 24D, first free-run condition; $\mathrm{DD} / \mathrm{NQ}(-1,-2,-3) 12 \mathrm{~N}: 12 \mathrm{Q}$ cycle separated into three sections: first, second, third; DD2-24D, final freerun condition. 
Fig. $\quad 1(a, b, c)$. standard raster plot from experiment 1 of drinking, feeding, and LMA, respectively (rat \#2). Activity from each day is shown by vertical tick marks plotted horizontally with respect to time of day in hours. For optimum rhythm visualization the record has been double plotted. The light entrainment period is designated by LD; first freerun condition is designated by DD1; sound application (DD N/Q) epoch is represented by the boxed areas; and the final freerun is designated by DD2 . Dotted horizontal lines (Fig. 1b) represent blockage of the feed tray sensors.
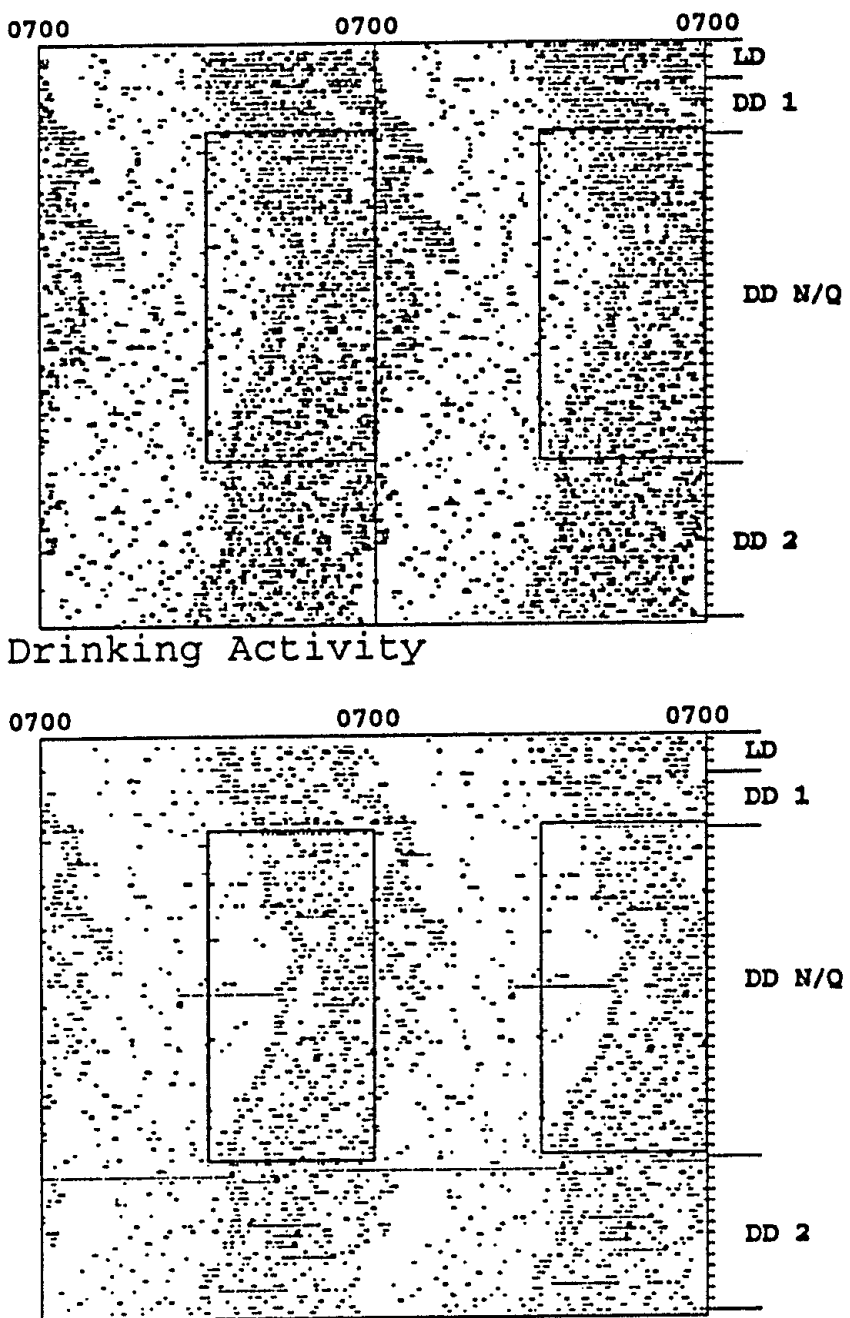

(b)

Feeding Activity

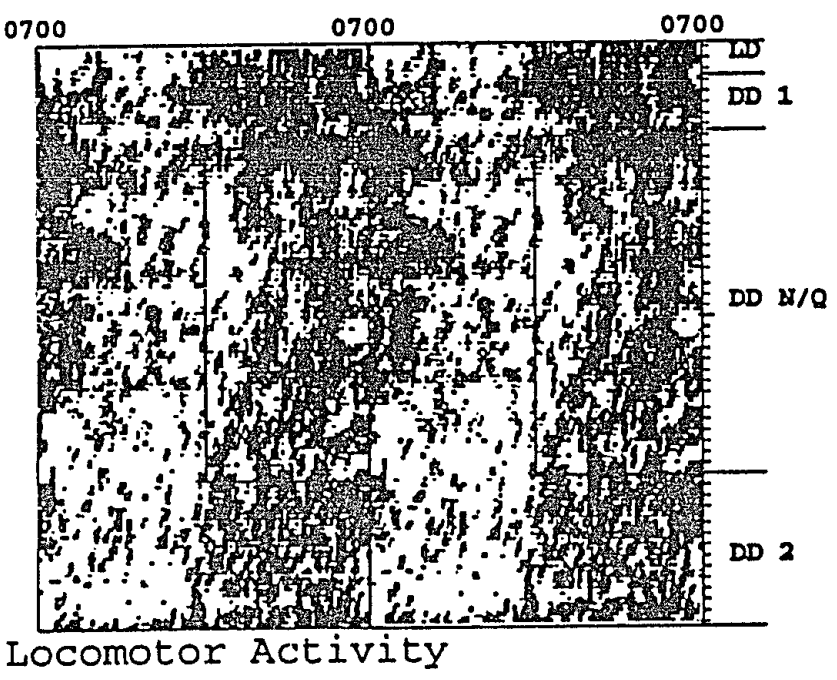


Tau (Drink): Before $(D D-1)$ \& after (DD-2) sound stimulus

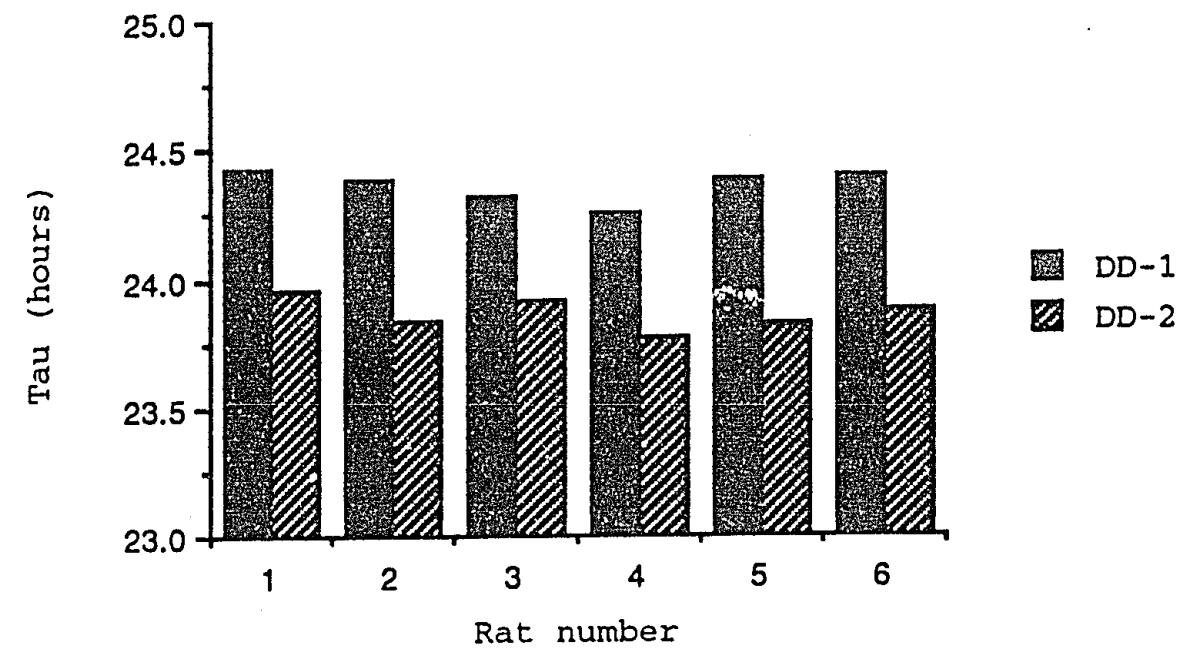

(a)

Tau (Feed): Before (DD-1) \& after (DD-2) sound stimulus

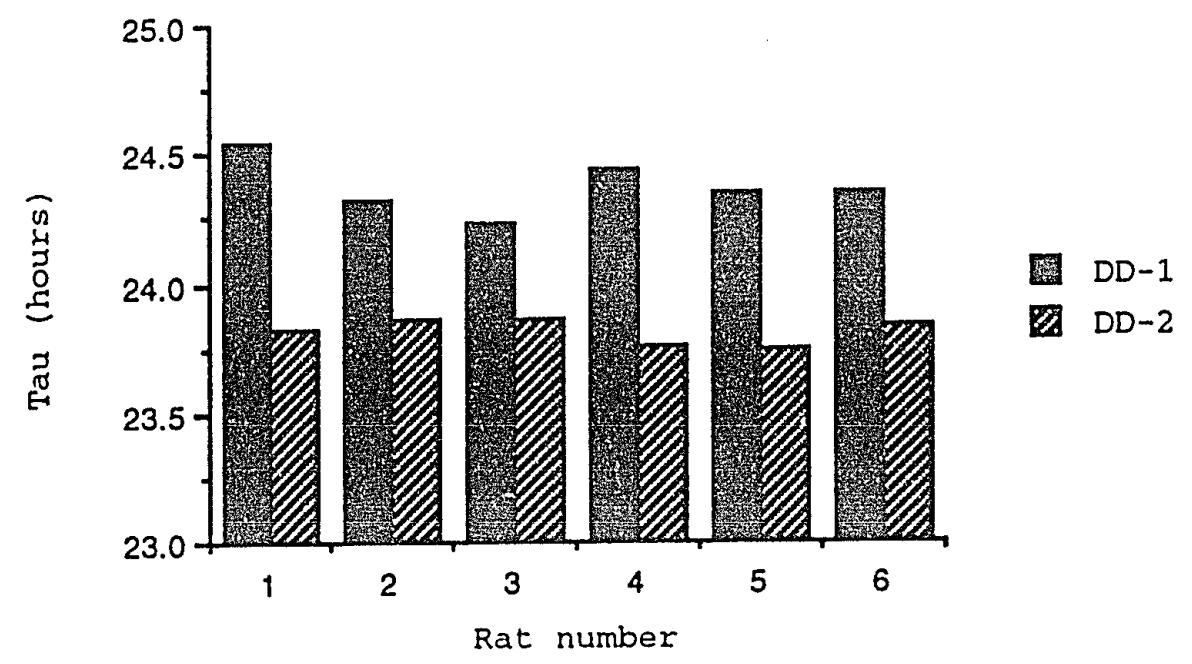

(b)

Figure $2(a, b)$. Histogram of estimated periods obtained from each rat in experiment 1 before and after the sound regimen was administered. (a) drinking data, (b) feeding data. 

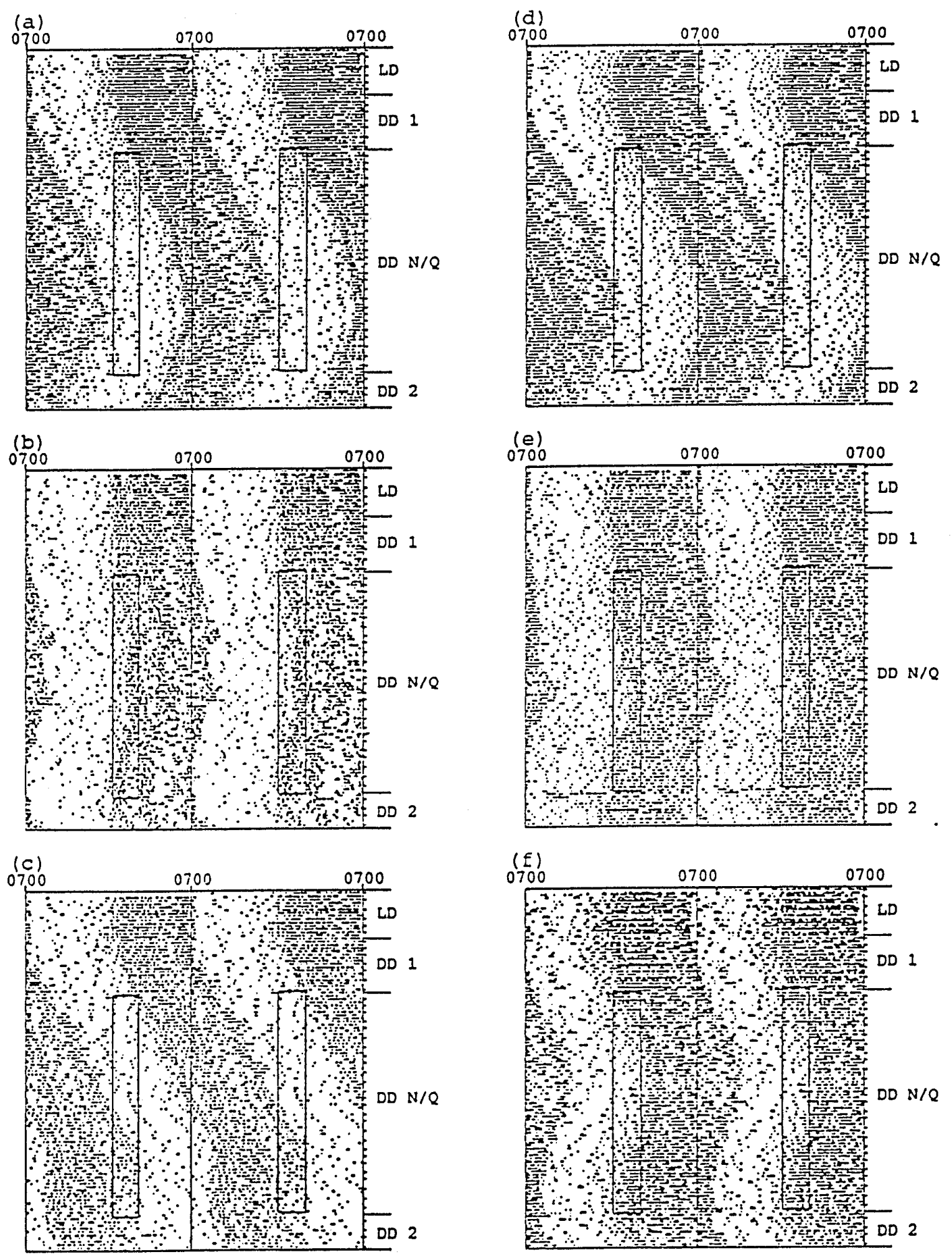

Figure $3 a-f$. Double plotted actograms of experiment 2 drinking activity for all six rats. Boxed areas indicate when sound was on. 

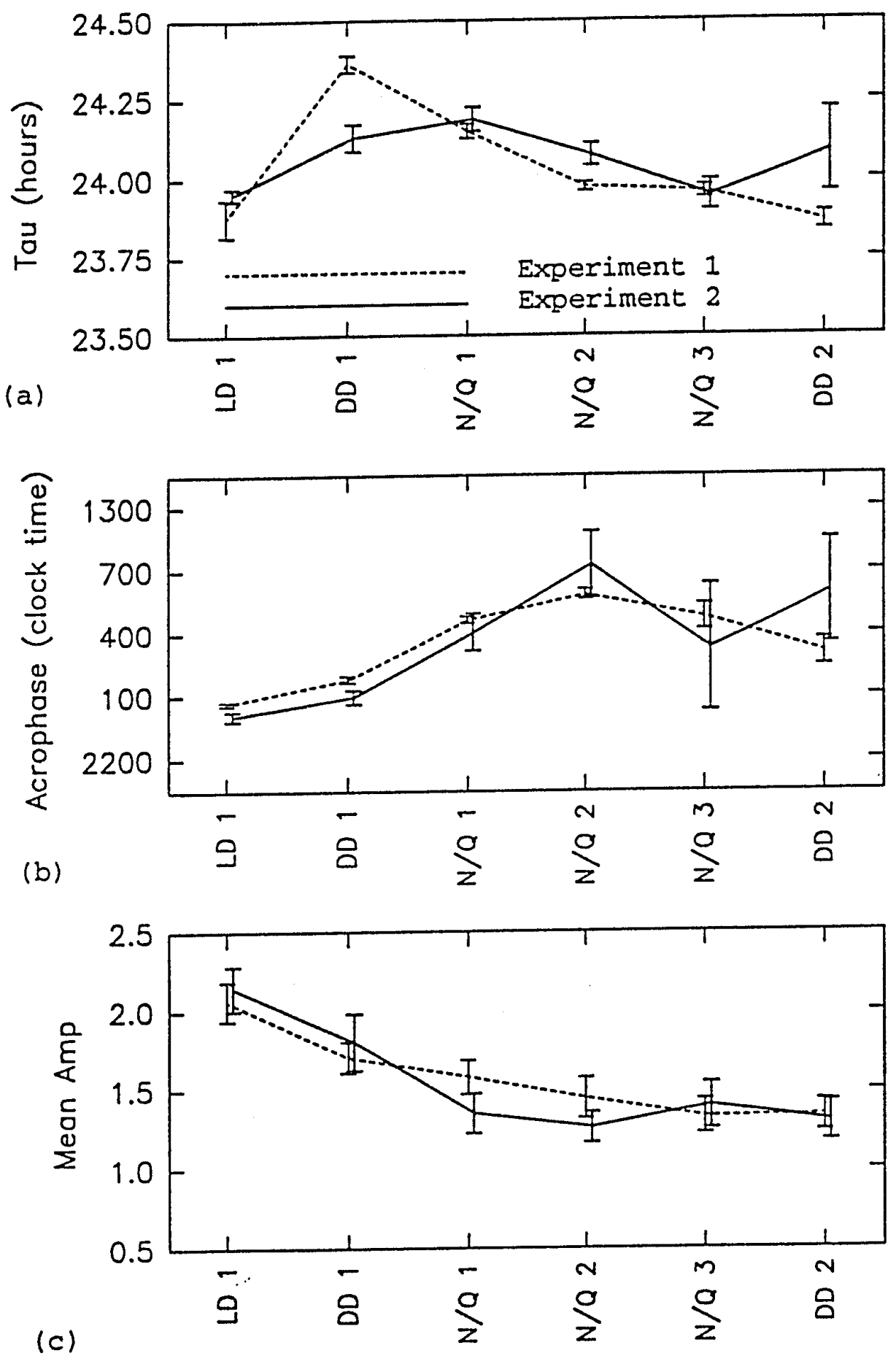

Figure 4. Comparisons between experiments 1 and 2 of mean and standard error of drinking activity values during each experimental condition. (a) Tau (hours), (b) acrophase (clock time), (c) amplitude (number of drinking events per 1 hour bin). Dashed line represents experiment 1 . Solid line indicates Experiment 2 . 


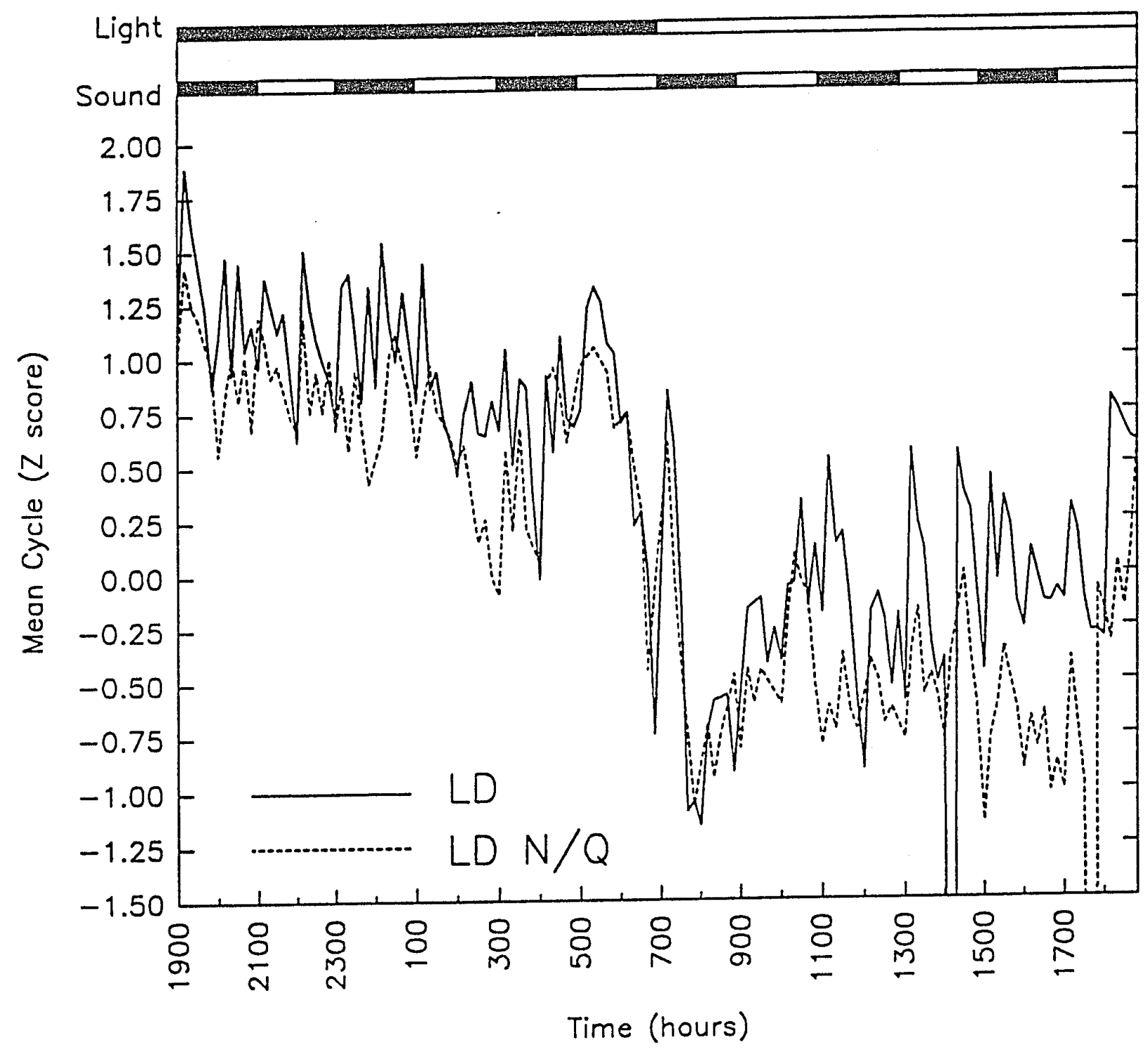

Figure 5. Experiment 3 mean cycle of LMA. Bars indicate light and sound schedules. Lights off denoted by dark bar. Sound on denoted by dark bar. 


\section{DISCUSSION}

our findings indicate that sound applied periodically can influence the circadian timing system of the Sprague-Dawley rat. In response to the imposed sound cycles, the circadian rhythm of activity/behavior expressed initial instability in period and phase (Experiment 1) followed by a shortened period; some periods measuring $<24 \mathrm{~h}$.

Upon release from the sound regimen back to free-running conditions all six rats in experiment 1 and two rats from experiment 2 continued to display periods of $<24 \mathrm{~h}$. This continuation of shortened periods following the removal of the stimulus supports the notion that the sound cycle disturbed the timing process of the underlying circadian pacemaker causing aftereffects in the circadian rhythm (Pittendrigh, 1960).

Experiment 3 was performed to determine if the observed shifts in phase and period were expressions of a masking effect in response to the applied sound. This experiment showed no significant indication of the presence of this type of effect. Moreover, it can be argued that if the endogenous pacemaker remained unaltered by the noise, upon removal of the sound the rhythm would resume its previously established free-running period length $(>24 \mathrm{~h})$ from the phase predicted by the endogenous clock and not by the environmental cycle. It did not.

The amplitude of the rhythms in the entrainment experiments 
declined at the same rates over the first freerun epoch (Fig. 4C). During the sound regimen and thereafter, however, the decrease of the amplitude did not remain constant. Speculatively, this could be due to some interactive property of sound on the strength of the rhythm. However, this cannot be conclusively explained until an experiment is performed to show the change in amplitude of the rhythm as a function of time.

An intriguing aspect of the results is that the period length shortened to $<24 \mathrm{~h}$ in 8 out of 12 rats exposed to the exogenous sound stimulus. Normally, Sprague-Dawley rats only show a Tau of $<24 \mathrm{~h}$ as an aftereffect to short photoperiods (Stephan, 1983). Wistar rats, which also normally freerun with periods of $>24 \mathrm{~h}$, have also been shown to express profound decreases in their freerunning period $(<24 \mathrm{~h})$ in response to intercerebral ventricular administrations of serotonergic agonists (Edgar et al., 1993). In addition to these findings, it has been shown that auditory stimulation leads to excitation, without habituation, of serotonergic cells in the Raphe Nucleus in cats (Rasmusson, 1984, 1986). If this information can be extrapolated to the rat, a possible neuromodulatory mechanism can be proposed: sound stimulation excites serotonergic cells in the Raphe Nuclei which sends ascending, efferent projections to the SCN (steinbusch, 1981) thus providing information to the primary circadian pacemaker. This proposed hypothesis is not specific to sound (e.g. several investigators suspect serotonin mediation of exercisedependent non-photic zeitgebers (Edgar et al., 1993)), but 
provides a possible mechanism consistent with Mrosovsky's (1988) proposed hypothesis for a non-photic "non-specific, arousal oscillator" which may influence the primary photic oscillator. Regardless of the actual mechanism, it is apparent that the circadian rhythm periods of the overt behaviors change when subjected to a temporal acoustic disturbance.

In this study, noise cycles did not meet all four standard criteria for entrainment (Moore-Ede et al., 1982). First, the overt rhythms must show a free-running period, independent of the environment, before the putative entrainer is administered and again after the temporal cue is removed. Second, in conjunction with the stimulus, the period of the monitored rhythm must adjust to become equal to that of the stimulus. Third, a stable phase relationship must be demonstrated. That is, the phase angle of the rhythm must be reproducible and constant in relationship to the timing of the zeitgeber. And finally, the zeitgeber must show phase control until it is removed. Once removed, the rhythm should begin free-running from the time in which it was being held.

In the first experiment of the entrainment study the majority of animals showed a decrease followed by an increase in Tau during the first 20 days of the sound epoch. Though "entrainment" was not evident, the resulting "scalloping" in the rhythm may indicate relative coordination (Pittendrigh \& Daan, 1976). The design of experiment 2 was based on: 1) the findings of Menaker and Eskin (1966) which indicated that noise entrainment 
was best achieved when the zeitgeber was presented at or near the onset of the animal's activity, and 2 ) information provided by the non-photic phase response curve (PRC) proposed by Mrosovsky (1988). According to this PRC, a zeitgeber applied between CT 10 and CT 14 would not allow for entrainment until the free-running rhythm advanced, or delayed, sufficiently to coincide with a position on the PRC that would result in rhythm phase control. Experiment 2 showed transient shifts in Tau late in the sound epoch but did not produce a "scalloping" of the rhythm as observed in experiment 1 . This could possibly be a function of zeitgeber duration. The $12 \mathrm{~h}$ sound application interacts with more phases of the animal's rhythm, thus producing a mixed combination of phase advances and delays. The $4 \mathrm{~h}$ sound application interacts with a smaller portion of the animal's rhythm, possibly accounting for the smaller net changes in phase and lack of scalloping in the data. Alternatively, the strength of the zeitgeber during the second experiment of the entrainment study may have been weaker due to the shorter stimulus duration.

The inability to show white noise-dependent entrainment in rats is inconsistent with the results of related studies performed using birds. For example, Siskins (Carduelis spinus), Serins (Serinus serinus), and House sparrows (Passer domesticus) were able to entrain to species-specific song cycles (Gwinner, 1966; Menaker \& Eskin, 1966; Reebs, 1989). In a study using greenfinches (Carduelis chloris) and chaffinches (Fringilla coelebs), Lohman \& Enright (1967) removed the "social" aspect of 
the acoustical zeitgeber by replacing the species-specific song cycles with cycles of continuous and intermittent noise produced by electrical buzzers and frequency generators. These birds showed weak entrainment to both types of sound stimuli. Thus, it is not the content of the sound stimulus that is crucial for entrainment; rather it is the temporal stimulation of the auditory pathways which is the crucial factor. Since our study did not demonstrate entrainment of rat circadian rhythms to electrically generated white noise, it is plausible that the ability to synchronize to general sound stimulation is specific to birds. Birds released from sound entrainment consistently demonstrate lengthening of Tau (Menaker \& Eskin, 1966; Reebs, 1989). In contrast, Tau continued to shorten after the stimulus was removed in our rats. It is likely that these period effects reflect latent influences of the preceding periodic stimuli although additional studies are needed for a definitive conclusion.

Reebs (1989) has reported an "acoustical" PRC for birds (Passer domesticus) using single, $2 \mathrm{~h}$ "pulses" of conspecific vocalization. That PRC suggests minimal changes in phase between CT 0 and CT 18 and suggests that advances occur between CT 18 and CT 24. Unfortunately, however, that PRC lacks sufficient data to be unequivocal.

only a few studies have addressed acoustical entrainment using mammals (Meyer, 1968; Randall et al., 1990; Sulzman et al., 1977). Sulzman et al.(1977) examined the ability of $12 \mathrm{~h}$ 
intermittent (2 min. ON:13 min. OFF) white noise to entrain the circadian rhythm of the squirrel monkey (Saimiri sciureus). Our first experiment (Exp. 1) was designed similarly to that of Sulzman et al., except that they used diurnal squirrel monkeys (Saimiri sciureus) which were held in constant light and subjected to a 10 day sound epoch. Acoustical entrainment was not observed in the squirrel monkeys, although one of the three experiments showed a shortening of Tau amongst the animals (Sulzman et al., 1977). Thus it would appear that nocturnal and diurnal mammals may respond similarly to periodic acoustical stimuli.

Randall et al. (1990) studied sound entrainment in cats. They subjected 4 individually isolated cats to an 8 hour recording of cat colony sounds during maintenance. In response to this daily stimulus, 3 of the 4 cats showed "weak" entrainment of their locomotor activity rhythms. The fourth cat demonstrated relative coordination. All cats showed immediate phase control when the sound stimulus onset corresponded with the activity onset. Though our study did not show phase control when the stimulus was presented during the activity onset, the Randall et al. (1990) findings support those reported by Menaker and Eskin (1966), specifically that entrainment occurred more readily when the onset of the sound stimulus corresponded with the onset of activity. The lack of period stability during the course of this study need not be attributed to the effects of sound alone. To the best of our knowledge, the longest freerun study performed on spragueDawley rats in constant darkness was 44 days which resulted in an. 
average Tau greater than $24 \mathrm{~h}$ (Stephan, 1983). It is not clear whether Tau remains stable beyond six weeks. Since it has been established that aging tends to shorten circadian free-running periods (Pittendrigh \& Daan, 1974) and our animals were maintained in constant dark (DD) for 110 days, additional work may be necessary to differentiate longitudinal study effects from the effects of periodic acoustical stimuli.

In summary, we have found that temporal noise disturbances can influence the circadian pacemaker in the rat. Changes in phase in response to the noise stimulation ultimately resulted in profound rhythm variability and decreases in Tau, the latter persisted after the stimulus was removed. 


\section{REFERENCES}

1. Aschoff, J. 1960. Exogenous and Endogenous Components in Circadian Rhythms. Cold Springs Harbor Symp. Quant. Biol. 25:11-28.

2. Aschoff, J., M. Fatranska, H. Geidke. 1971. Human circadian rhythms in continuous darkness: entrainment by social cues. Science 171:213-215.

3. Aschoff, J., S. Daan, G. A. Groos (eds). 1982. Vertebrate Circadian systems: Structure and Physiolocy, springer Verlag, Berlin.

4. Aschoff, J., C. von Goetz, K. Honma. 1983. Restricted feeding in rats: effects of varying feeding cycles. Tierpsychol. 63:91-111.

5. Bloomfield, P. 1976. Fourier Analysis of Time Series: An Introduction. John Wiley \& Sons, Inc., New York

6. Borbély, A. A. 1982. Circadian and sleep-dependent Processes in sleep regulation, IN: Vertebrate Circadian Systems, Aschoff, J., Daan,S., G. Groos (eds), SpringerVerlag, Berlin. pp. 237-242.

7. Bruce, V. G. 1960. Environmental entrainment of circadian rhythms. Cold Springs Harbor Symp. Quant. Biol. 25:49-55.

8. Cleveland, W.S. 1979. Robust locally weighted regression and smoothing scatterplots. J. Am. Stat. AssoC. $14: 829-836$.

9. Chandreshekaran, M. K. 1982. Social cues and circadian rhythms. Current science. 51:158-167.

10. Crowley, M., J. Bovet. 1980. Social synchronization of circadian rhythms in Deer Mice (Peromyscus maniculatus). Behav. Ecol. Sociobiol. 7:99-105.

11. Czeisler, C. A., G. S. Richardson, R. M. Coleman, J. C. Zimmerman, M. C. Moore-Ede, E. D. Weitzman. 1981. Entrainment of human circadian rhythms by light-dark cycles: a reassessment. Photochem. Photobiol. 34:239247 .

12. Dowse, H. B., J. D. Palmer. 1969. Entrainment of circadian activity rhythms in mice by electrostatic 
fields. Nature 222:564-566.

13. Edgar, D. M., C. E. Martin, W. C. Dement. 1991. Activity feedback to the mammalian circadian pacemaker: influence on observed measures of rhythm period length. J. Biol. Rhythms. 6:185-199.

14. Edgar, D. M., J. D. Miller, R. A. Prosser, R. R. Dean, W. C. Dement. 1993. Serotonin and the mammalian circadian system: II. phase-shifting rat behavioral rhythms with serotonergic agonists. J. Biol. Rhythms. 8 : (in press).

15. Edmonds, S. C., N. T. Adler. 1977. Food and light as entrainers of circadian running activity in the rat. Physiol. and Behav. 18:915-919.

16. Erkert, H. G., B. Nagel, I. Stephani. 1986. Light and social effects on the free-running circadian activity rhythm in common marmosets (Callithrix jacchus; Primates): social masking, pseudo-splitting, and relative coordination. Behav. Ecol. Sociobiol. 18: $443-452$

17. Gwinner, E. 1966. Periodicity of a circadian rhythm in birds by species-specific song cycles. Experientia. $\underline{22}: 765-766$.

18. Hayden, P., R. G. Lindberg. 1969. Circadian rhythm in mammalian body temperature entrained by cyclic pressure changes. Science. 164:1288-1289.

19. Kleinknecht, S. 1985. Lack of social entrainment of free-running circadian activity rhythms in the Australian sugar glider (Petaurus brevis: Marsupialia). Behav. Ecol. Sociobiol. 16:189-193.

20. Lindberg, R. G., P. Hayden. 1974. Thermoperiodic entrainment of arousal from torpor in the little pocket mouse, Perognathus longimembris. Chronobiologia.1: .356-361.

21. Lohmann, M., J. T. Enright. 1967. The influence of mechanical noise on the activity rhythms of finches. Comp. Biochem. Physiol. 22:289-296.

22. Marimuthu, G., R. Subbaraj, M. K. Chandrashekaran. 1978. Social synchronization of the activity rhythm in a cave dwelling insectivorous bat. Naturwiss. 65:600.

23. Marimuthu, G., M. K. Chandrashekaran. 1979. Social synchronization of circadian rhythms in bats. 
Chronobiologia. 6:129.

24. Marimuthu, G., M. K. Chandrashekaran. 1981. Social chronobiology of a species of bat. Inter. $J$. Chronobiology. Z:88.

25. Meyer, A. 1968. Einfluß von Schall auf die tagesperiodische Aktivität des Goldhamsters. Naturwiss. 55:234-235.

26. Menaker, M., A. Eskin. 1966. Entrainment of circadian rhythms by sound in Passer domesticus. Science 23: 1579-1581.

27. Mistlberger, R. E., T. A. Houpt, M. C. Moore-Ede. 1990. Characteristics of food-entrained circadian rhythms in rats during long-term exposure to constant light. Chronobiology Inter. $7: 383-391$.

28. Moore-Ede, M. C., F. M. Sulzman, C. A. Fuller. 1982. The clocks that Time Us: Physiology of the circadian Timina System, Harvard Univ. Press, MA.

29. Mrosovsky, N. 1988. Phase response curves for social entrainment. J. Comp. Physiol. 162:35-46.

30. Pittendrigh, C.S. 1960. Circadian rhythms and the circadian organization of living systems. Cold Springs Harbor Symp. Quant. Biol. 25:159-182.

31 Pittendrigh, C.S., S. Daan. 1974. Circadian oscillations in rodents: a systematic increase of their frequency with age. Science. 186:548-550.

32. Pittendrigh, C.S., S. Daan. 1976. A functional analysis of circadian pacemakers in nocturnal rodents. IV. Entrainment: Pacemaker as clock. J. Comp. Physiol., 106:291-331.

33. Randall, W. J., T. Cunningham, S. Randall. 1990. Sounds from an animal colony entrain a circadian rhythm in the cat, Felis catus L. J. Interdiscipl. Cycle Res. 21: $51-64$.

34. Rasmusson, K., J. Heym, B. L. Jacobs. 1984. Activity of serotonin-containing neurons in nucleus centralis superior of freely moving cats. Exp. Neurol. 83:302317.

35. Rasmusson, K., R. E. Strecker, B. L. Jacobs. 1986. Single unit response of noradrenergic, serotonergic and dopaminergic neurons in freely moving cats to simple 
sensory stimuli. Brain Res. 369:336-340.

36. Reebs, S. G. 1989. Acoustical entrainment of circadian activity rhythms in House sparrows: constant light is not necessary. Ethology 80:172-181.

37. Rusak, B.J.,1988. Daily hoarding opportunity entrains the pacemaker for hamster activity rhythms $J$. Comp. Phys. A. 164:165-171.

38. Sing, H. C., D. P. Redmond, F. W. Hegge. 1986. Multiple complex demodulation: a method for rhythmic analysis of physiological and biological data, IN: proceedings in the Fourth Annual symposium on computer Applications in Medical Care, IEEE, New York, pp. 151158 .

39. Steinbusch, H.W.M. 1981. Distribution of serotoninimmunoreactivity in the central nervous system of the rat--cell bodies and terminals. Neuroscience 4:557618 .

40. Stephan, F. K. 1983. Circadian rhythms in the rat: constant darkness, entrainment to $\mathrm{T}$ cycles and to skeleton photoperiods. Physiol. \& Behav. 30:451-462.

41. Sulzman, F. M., C. A. Fuller, M. C. Moore-Ede. 1977. Environmental synchronizers of squirrel monkey circadian rhythms. J. Appl. Physiol. 43:795-800.

42. Sweeney, B., J.W. Hastings. 1960. Effects of Temperature upon diurnal rhythms. Cold Springs Harbor Symp. Quant. Biol. 25:87-104.

43. Tukey, J.W. 1977. Exploratory Data Analysis. AddisonWesley, Reading, MA.

44. Wever, R. 1979. The Circadian System of Man: Results of Experiments under Temporal Isolation, SpringerVerlag, Berlin. pp. 150-159.

45. Winget, C. M., C. W. DeRoshia, K. H. Ogawa, D. C. Holley. 1989. Significance of light and social cues in the maintenance of temporal organization in man. The Physiologist 32 (Suppl.) : S94-596.

46. Zar, J. H. 1984. Biostatistical Analysis, Second Edition, Prentice Hall, New Jersey. 